OPEN ACCESS

Edited by:

Xiaotong Wang,

Ludong University, China

Reviewed by:

Yuewen Deng,

Guangdong Ocean University, China

Keren Cheng,

University of Texas at San Antonio,

United States

Xian De Liu,

Jimei University, China

*Correspondence:

Yong-ren Li

lyr1018@163.com

tThese authors have contributed equally to this work

Specialty section:

This article was submitted to

Aquatic Physiology,

a section of the journal

Frontiers in Marine Science

Received: 13 January 2021

Accepted: 17 February 2021

Published: 10 March 2021

Citation:

Li Y, Zhang B, Liang S and Guo Y (2021) Meiotic Spindle Formation

Following Inhibition of First Polar Body Formation in the Zhikong Scallop

(Chlamys farreri Jones et Preston).

Front. Mar. Sci. 8:653000.

doi: 10.3389/fmars.2021.653000

\section{Meiotic Spindle Formation Following Inhibition of First Polar Body Formation in the Zhikong Scallop (Chlamys farreri Jones et Preston)}

\author{
Yongren Li ${ }^{1 * t}$, Baolu Zhang ${ }^{2 t}$, Shuang Liang ${ }^{1}$ and Yongjun Guo ${ }^{1}$ \\ ${ }^{1}$ Key Laboratory for Aquatic Ecology and Aquaculture of Tianjin, Department of Fisheries Science, Tianjin Agricultural \\ University, Tianjin, China, ${ }^{2}$ Oceanic Consultation Center, Ministry of Natural Resources of the People's Republic of China, \\ Beijing, China
}

Fertilized Zhikong scallop (Chlamys farreri) eggs were treated with cytochalasin B (CB $0.5 \mathrm{mg} / \mathrm{L}$ ) at $14-15 \mathrm{~min}$ postfertilization to inhibit first polar body formation. The eggs were then stained with fluorescein isothiocyanate (FITC) -anti- $\alpha$-tubulin and propidium iodide (PI) to examine their microtubule patterns and chromosome, respectively. Fluorescent microscope observations of treated eggs sampled every 23 min during meiotic maturation revealed meiotic apparatus assembly and correlated chromosome segregation. In CB-treated groups, meiosis I proceeded normally and produced two groups of dyads, with 19 in each group. Both dyad groups were retained in the eggs as they entered meiosis II. Two, three, or four asters (centrosome with microtubules around it) in meiosis II rearranged the spindle in several patterns: bipolar $[24.0 \pm 4.1 \mu \mathrm{m}$ (long axis) $\times 18.3 \pm 4.1 \mu \mathrm{m}$ (diameter: metaphase plate)], tripolar $(18.6 \pm 3.9 \mu \mathrm{m} \times 9.9 \pm 1.3 \mu \mathrm{m})$, separated bipolar $(18.3 \pm 2.8 \mu \mathrm{m} \times 11.2 \pm 1.8 \mu \mathrm{m})$, and other unclassified spindle patterns. Corresponding chromosome segregation, including bipolar (18.9\%), tripolar (38.9\%), double bipolar (16.5\%), and unclassified (25.6\%), was observed during meiosis II in CB-treated eggs. The data indicated that chromosome segregation patterns determined by spindle patterns were critically influenced by the number of centrosomes in meiosis II eggs following inhibition of polar body 1 (PB1) formation with CB.

Keywords: Chlamys farreri, polyploid, meiotic spindle, chromosome segregation, cytochalasin B

\section{INTRODUCTION}

Chromosome manipulation of marine bivalves has received considerable research interest. Following inhibition of polar body 1 (PB1) formation, tetraploids have been reported in Manila clam Ruditapes philippenarum (Li et al., 2017), Pacific abalone Haliotis discus hannai (Arai et al., 1986), American oyster Crassostrea virginica (Stanly et al., 1981), Pacific oyster Crassostrea gigas (Stephens and Dowing, 1988; McCombie et al., 2005), Hong Kong oyster Crassostrea hongkongensis (Qin et al., 2018), pearl oyster Pinctada martensii (He et al., 2000), and other mollusk species (Yamamoto and Sugawara, 1988; Guo and Allen, 1994; Yang et al., 2000a; Tan et al., 2017). When PB1 formation was blocked in C. gigas zygotes, observations of chromosome segregation 
revealed possible mechanisms of different ploidy formation (Guo et al., 1992). Previous studies of the effect of blocking PB1 formation on the subsequent chromosome segregation in fertilized eggs from triploid C. gigas revealed various segregation patterns during meiosis II, including tripolar segregation, united bipolar segregation, and separated bipolar segregation (Que et al., 1997). Yang et al. (2000b) used a hematoxylin staining method to analyze chromosome segregation in fertilized Zhikong scallop (Chlamys farreri) eggs following blocking of PB1 formation by cytochalasin B (CB). They hypothesized that centrosomes and spindles might play an important role in meiosis. However, this hypothesis was not supported by cytological observations based on immunofluorescent staining (Que et al., 1997).

In this study, a new fluorescent staining method for eggs of marine bivalves was established using fluorescein isothiocyanate (FITC)-anti- $\alpha$-tubulin and propidium iodide (PI); these agents allow for observation of spindles and chromosomes, respectively. Asters, which are the meiotic apparatuses that assemble spindles and coordinate chromosome segregation, were observed in fertilized eggs from normal diploids when PB1 formation was blocked with CB. The probable cytological explanation for the production of tetraploids was documented, and the hypothesis that centrosomes and spindles are the primary factors controlling patterns of chromosome segregation was tested.

\section{MATERIALS AND METHODS}

\section{Gametes}

In this study, 2-year-old Zhikong scallop breeders were collected from Qingdao, Shandong, China. The scallops were conditioned indoors until the gonads reached maturity. Gametes were obtained from several parent scallops through natural spawning. Eggs were filtered through a $60 \mu \mathrm{m}$ Nytex screen to remove gonadal tissue and debris, and washed twice in sea water, then re-suspended in $4 \mathrm{~L}$ seawater at $20^{\circ} \mathrm{C}$, at which time they were ready for fertilization. Sperm were filtered through a $25 \mu \mathrm{m}$ screen to remove tissue debris. For fertilization, sperm were added to the egg suspension at a final density of 5-7 sperm per egg. All fertilizations and incubations were conducted at $20^{\circ} \mathrm{C}$ in filtered seawater.

\section{Cytochalasin B Treatment}

Two experimental groups were established: In the first group, fertilized eggs were allowed to develop normally, and these served as the control. In the second group, fertilized eggs were treated with $0.5 \mathrm{mg} / \mathrm{l} \mathrm{CB}$ in $0.05 \%$ dimethylsulfoxide (DMSO), and these served as the treated group. In the treated group, CB was added to egg suspensions just before insemination, immediately following PB1 formation, or during the PB1 forming period [14-15 min post-fertilization (PF), at which time PB1 had occurred in the control]. The CB treatment was stopped as soon as PB2 was observed in the control group (33-35 min PF). Zygotes were washed twice and suspended in fresh seawater. The fertilized eggs in the control group were suspended in DMSO at a final concentration of $0.5 \mathrm{~g} / \mathrm{L}$. The experiment was repeated twice using different parent scallops.

\section{Immunofluorescent Staining}

Fertilized eggs from both the control and CB-treated groups were sampled every 2-3 min during development until $40 \mathrm{~min}$ PF. Samples were directly fixed using formaldehyde fixative [4 g polyformaldehyde dissolved in $100 \mathrm{~mL} 0.2 \mathrm{~mol} / \mathrm{L}$ phosphate buffered saline (PBS)], which was changed twice, and the samples were stored at $4^{\circ} \mathrm{C}$ for analysis.

Specimens were permeabilized with $0.5 \%$ Triton X-100 for $40 \mathrm{~min}$ and then washed twice in PBS and suspended into RNase solution for $60 \mathrm{~min}$ at a final concentration of $200 \mu \mathrm{g} / \mathrm{ml}$. Specimens were incubated in $3 \%$ bovine serum albumin (BSA) in PBS for $1 \mathrm{~h}$, followed by $1 \mathrm{~h}$ incubation in FITC-anti- $\alpha$-tubulin (1/50 dilution, Sigma). Specimens were washed twice in PBS, incubated in PI (10 $\mu \mathrm{g}$ in $1 \mathrm{~mL}$ PBS, Sigma) for $10 \mathrm{~min}$, rinsed in $\mathrm{PBS}$, and then transferred into a drop of mounting medium (2\% DABCO in $90 \%$ glycerol in $0.1 \mathrm{~mol} / \mathrm{L}$ Tris-HCl, $\mathrm{pH} 7.4$ ) on clean slides. A clean cover glass was placed gently on the sample.

Slides were examined under a Nikon Eclipse 50i fluorescent microscope. Photographs were taken using a CCD system. The length and width of the first and second meiotic spindles were measured for 50 samples. Spindle measurement data are presented as means $\pm \mathrm{SD}$.

\section{RESULTS}

Initially, normal (i.e., control) cells were observed in the metaphase of meiosis I with chromosomes anchored in the metaphase plate. The long axis of the first meiotic spindle was $27.2 \pm 4.4 \mu \mathrm{m}$, and its diameter at the metaphase plate measured $14.8 \pm 2.5 \mu \mathrm{m}$. In all specimens examined, the long axis of the spindle was positioned normal to the egg surface.

In the control group, the chromosomes began to segregate at about 9-10 min PF and then divided into two groups. One group of chromosomes condensed and was released as PB1. This was followed by reorganization of the second group of chromosomes onto the metaphase plate of the spindle in meiosis II. The size of the second meiotic spindle, including both the long axis $(19.7 \pm 3.7 \mu \mathrm{m})$ and diameter $(9.4 \pm 2.9 \mu \mathrm{m})$, was approximately $2 / 3$ that of the first group of chromosomes that produced PB1 during meiosis I.

In the CB-treated group, meiotic apparatuses and chromosome segregation during meiosis I were similar to those in the control group, except PB1 formation was blocked in the majority of fertilized eggs. However, in the metaphase and anaphase of meiosis II, the meiotic apparatuses and chromosome segregation pattern differed greatly from those of the control group. Four typical spindle patterns were observed (bipolar, tripolar, separated bipolar, and unclassified), and they corresponded to four chromosome segregation patterns (bipolar, tripolar, separated bipolar, and unclassified).

\section{Bipolar Segregation}

The bipolar spindle (Figure 1A) formed in prophase II and was followed by reorganization of the chromosomes onto the metaphase plate of the spindle. All chromosomes segregated in a bipolar pattern in the anaphase, just like normal meiosis II, and 


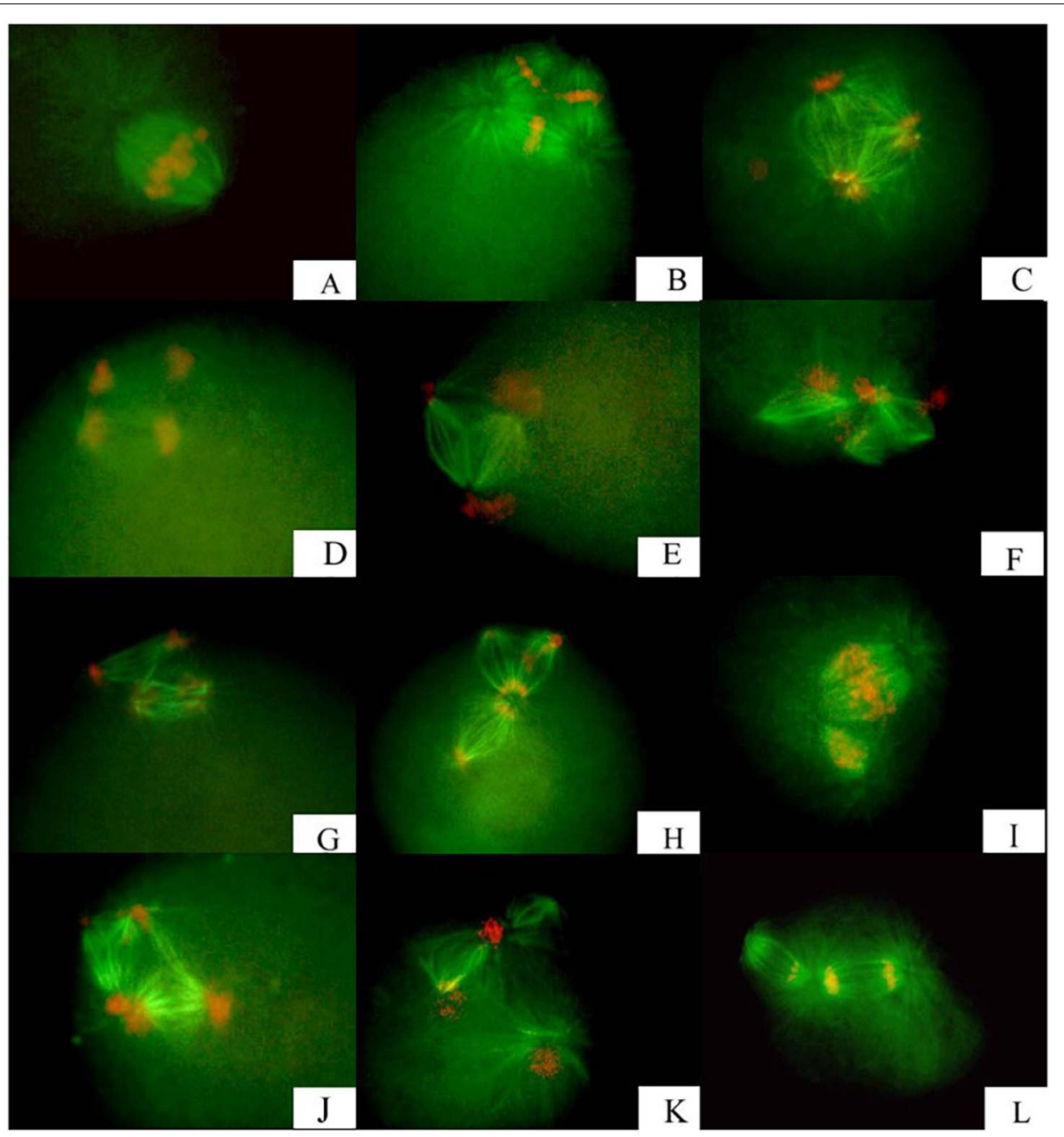

FIGURE 1 | Meiotic apparatuses assembling and correlated chromosome segregation observed in fertilized eggs from triploid Zhikong scallop, following inhibition of polar body I. (A) Bipolar segregation with bipolar spindles. (B,C) Tripolar segregation with tripolar spindles. (D,E) Separated bipolar segregation with two bipolar spindles. (F-L) Unclassified segregation with several types of spindles at low frequency.

they divided into two groups of chromosomes. One group was released as PB2 after CB treatment. The spindles, especially those along the metaphase plate, were a larger size than those in normal meiosis II (Table 1) and they had more polar microtubules. In

TABLE 1 | Spindle patterns and corresponding chromosome segregation patterns in meiosis II eggs from C. farreri following inhibition of PB1 with CB.

\begin{tabular}{lclc}
\hline $\begin{array}{l}\text { Segregation } \\
\text { patterns }\end{array}$ & Percentage & Spindle patterns & $\begin{array}{c}\text { Spindle size (long } \\
\text { axis } \times \text { diameter) }\end{array}$ \\
\hline Bipolar & $18.9 \%$ & Bipolar spindles & $\begin{array}{c}24.0 \pm 4.1 \mu \mathrm{m} \times \\
18.3 \pm 4.1 \mu \mathrm{m}\end{array}$ \\
Tripolar & $38.9 \%$ & Tripolar spindles & $\begin{array}{c}18.6 \pm 3.9 \mu \mathrm{m} \times \\
9.9 \pm 1.3 \mu \mathrm{m}\end{array}$ \\
$\begin{array}{l}\text { Separated } \\
\text { bipolar }\end{array}$ & $16.5 \%$ & Two bipolar spindles & $18.3 \pm 2.8 \mu \mathrm{m} \times 11.2$ \\
Unclassified & $25.6 \%$ & $\begin{array}{l}\text { Several types of } \\
\text { spindles at low }\end{array}$ & \\
& & & \\
& & & \\
& & &
\end{tabular}

all specimens examined, the spindle was positioned near the membrane with the long axis perpendicular to the cell surface.

\section{Tripolar Segregation}

All chromosomes were reorganized onto three metaphase plates of the tripolar spindle in metaphase II (Figure 1B). Chromosomes in each plate segregated in a bipolar pattern in the anaphase and eventually were concentrated at three poles, with a random number of chromosomes at each pole (Figure 1C). The spindle was shaped like an equilateral triangle, with the length of each side approximately equal to the long axis of spindles that formed at metaphase II in untreated eggs (Table 1 and Figures 1B,C).

\section{Separated Bipolar Segregation}

For separated bipolar segregation, two spindles were present near the surface of the zygote in metaphase II, with two groups of chromosomes in two metaphase plates, respectively. The 
chromosomes were segregated in a normal bipolar pattern in anaphase II (Figures 1D,E). The size and shape of the two spindles in a given egg were similar. However, the length was appreciably smaller and the diameter at the metaphase plate was appreciably larger than those of spindles that formed at metaphase II in untreated eggs (Table 1).

\section{Unclassified}

In addition to the above segregation patterns, other unclassified patterns were observed, but these segregation patterns were not in disorder. Spindles observed in these eggs were present in special structures and were not similar (Figures 1F-L). Examples include three spindles joined together by two communal centrosomes (Figure 1F); three spindles joined together by only one communal centrosome (Figure 1F); two spindles of different sizes joined together by one communal centrosome (Figures 1I,L); and tetrapolar spindles with four centrosomes in the shape of a square (Figure 1J). Generally, dissociative asters were observed in several zygotes except for zygotes in which the spindle inside had four poles (Figure 1K). Some dissociative asters organized chromosomes onto the asteral microtubules.

The frequencies of the three segregation patterns in meiosis II were calculated based on observations of the meiotic apparatuses of fertilized eggs for which PB1 had been blocked (Table 1). Table 1 also shows the spindle measurements from meiosis II.

\section{Asters}

Aster number was counted when chromosome segregation in eggs treated with CB was examined. Zygotes of treated groups had two, three, or four asters (Table 2), and several zygotes (2-3\%) contained dissociative asters.

\section{DISCUSSION}

\section{Double Staining Method}

Immunofluorescence is used to study microtubule skeletal structure, spindles, and centrosomes in the fertilized eggs of many animals (Zhu et al., 2008). We developed a new modified immunofluorescent double staining method using FITC-anti- $\alpha$ tubulin and PI to observe the assembly of meiotic apparatuses and the correlated chromosome segregation in fertilized $C$. farreri eggs. This method proved to be effective for simultaneous observation of spindles and chromosomes in C. farreri eggs and should be applicable to other marine bivalves. It offers

TABLE 2 | Percentage of fertilized $C$. farreri eggs with a specific number of asters in meiosis II following inhibition of PB1 with CB.

\begin{tabular}{lcccc}
\hline Replicate (number) & \multicolumn{4}{c}{ Number of asters } \\
\cline { 2 - 5 } & $\mathbf{n}$ & $\mathbf{2}$ & $\mathbf{3}$ & $\mathbf{4}$ \\
\hline 1 & 173 & $19.1 \%$ & $39.3 \%$ & $41.6 \%$ \\
2 & 171 & $12.7 \%$ & $42.3 \%$ & $45.1 \%$ \\
Average & & $15.9 \%$ & $40.8 \%$ & $43.3 \%$
\end{tabular}

both simplicity in slide preparation and high imaging definition (Li et al., 2009).

In a study of morphogenesis of maternal and paternal genomes in Pacific oyster eggs, Longo et al. (1993) used 7aminoactinomycia $\mathrm{D}(7-\mathrm{AAD})$ and anti- $\beta$-tubulin to examine DNA; however, it is difficult to examine spindle formation and chromosome segregation patterns in one zygote because of unsynchronous spindles. Komaru et al. (1990) and Aya et al. (2017) used 4'6-diamidino-2-phenylindole (DAPI) to study chromosomes of pearl oysters. PI used in this study produced high luminance but poor contrast. Consequently, we used BSA and RNase solution to decompose RNA and enhance contrast. Compared with staining methods using orcein or hematoxylin, our new staining method allows observation of meiotic apparatuses, including spindles and chromosomes, but it is difficult to count the number of chromosomes due to low definition.

\section{Spindles and Chromosome Segregation}

Fan et al. (2002) reported that the meiotic cell cycle progression of rat oocytes is accompanied by extensive rearrangement of the microtubule network of the cell. The fertilized oocyte of C. elegans assembles two consecutive acentrosomal meiotic spindles that function to reduce the replicated maternal diploid set of chromosomes to a single-copy haploid set (Muller-Reichert et al., 2010). Our observations of meiotic apparatuses in Zhikong scallop zygotes with PB1 blocked illustrated that chromosome segregation patterns were determined by spindle patterns. Spindles with a given shape organized chromosomes according to their specific character. For example, when a tripolar spindle was present, all chromosomes were reorganized onto three metaphase plates during the metaphase and separated into six groups during anaphase II (Figures 1B,C). In the case of a separated bipolar spindle, two groups of chromosomes reorganized onto two metaphase plates of two separate spindles and separated into four groups during anaphase II (Figures 1D,E). In eggs from both Zhikong scallop and American oyster C. virginica (Hotta et al., 2017), the percentage of each chromosome segregation pattern calculated based on spindle type was approximately equal to that based on chromosome distribution.

Among the three main types of meiosis II spindles in C. farreri eggs with $\mathrm{PB} 1$ blocked by $\mathrm{CB}$ treatment, the bipolar spindle was the largest; it even had a greater diameter than that of normal meiosis I spindles at the metaphase plate. The number of chromosomes on the bipolar spindle was twice that of a normal bipolar spindle during meiosis II. Accordingly, it also was nearly twice the diameter at the metaphase plate. In contrast, the tripolar spindle, separated bipolar spindle, and normal spindle were similar in length and diameter at the metaphase plate during meiosis II (Table 1). However, the diameter at the metaphase plate of separated bipolar spindles was appreciably larger than that of bipolar spindles. We conclude that (1) the long axis in meiosis II was shorter than that in meiosis I regardless of the separation pattern.

Previous studies of the effect of blocking PB1 on subsequent chromosome segregation in diploid eggs using the squashing method revealed an unsynchronized segregation pattern. One 
group of dyads from meiosis I remained as sister chromatids, whereas the other group of dyads was divided and moved toward opposite poles (Yang et al., 2000a). In this study, we observed dissociative asters in several zygotes (1\%-2\%), and some dissociative asters organized chromosomes onto the asteral microtubules (Figure 1K). The method used in this study failed to reveal if the chromosomes fixed on dissociative asters were dyads.

\section{Centrioles and Spindles}

Centrioles are intimately involved in the formation of meiotic spindles in Mytilus edulis (Longo and Anderson, 1969), Spisula solidissima (Longo and Anderson, 1970), and mouse oocytes (Bennabi et al., 2018). The role of centrioles in establishing tripolar meiotic apparatuses was also suggested in a recent investigation of the effect of $\mathrm{CB}$ during meiotic maturation of diploid eggs from C. gigas (Longo et al., 1993). In mammals, centrioles in PB1 can divide normally under appropriate conditions (Fan, 1999). In addition, centrioles and associated proteins may regulate microtubule organization and spindle pole integrity in mouse oocytes (Yuan et al., 2009).

This study illustrated that the behavior of centrioles was ultimately responsible for the observed spindle and chromosome segregation patterns. Normally, the centrosome from sperm does not participate in meiosis (Sluder et al., 1993). With PB1 blocked in eggs, we observed that aster number ranged from two to four (Table 2) depending on whether the centrosomes replicated. Two asters resulted in bipolar spindles (Figure 1A); three asters resulted in tripolar spindles and some of the unclassified spindle patterns (Figures 1B,C,I,L), and four asters resulted in separated

\section{REFERENCES}

Arai, K., Naito, F., and Fujino, K. (1986). Triploidization of the Pacific abalone with temperature and pressure treatment. Bull. Jpn. Soc. Sci. Fish. 52, 417-422. doi: $10.2331 /$ suisan.52.417

Aya, I., Takahisa, K., and Yuichiro, K. (2017). A novel immunofluorescence method to visualize microtubules in the antiparallel overlaps of microtubule-plus ends in the anaphase and telophase midzone. Exp. Cell Res. 360, 347-357. doi: 10.1016/j.yexcr.2017.09.025

Bennabi, I., Queguiner, I., and Kolano, A. (2018). Shifting meiotic to mitotic spindle assembly in oocytes disrupts chromosome alignment. Embo Rep. 19, 368-381. doi: $10.15252 / \mathrm{embr} .201745225$

Fan, B. (1999). Oocyte meiotic maturation and development of parthenogenic and reconstituted embryos in mammals. J. Agric. Sci. 15, 116-126.

Fan, H., Tong, C., Li, S., Chen, D., and Sun, Q. (2002). Microtubule organization during in vitro development of rat oocytes. Prog. Biochem. Biophys. 29, 708713.

Guo, X., and Allen, S. (1994). Viable tetraploids in Pacific oyster Crassostrea gigas (Thunberg) produced by inhabiting polar body I in eggs from triploids. Mal. Mar. Biol. Biotech. 3, 42-50.

Guo, X., Hershberger, W., Cooper, K., and Chew, K. (1992). Genetic consequences of blocking polar body I with cytochalasin B in fertilized eggs of the Pacific oyster, Crassostrea gigas: II. Segregation of chromosomes. Biol. Bull. 183, 387393. doi: $10.2307 / 1542014$

He, M., Lin, Y., Shen, Q., Hu, J., and Jiang, W. (2000). Production of tetraploid pearl oyster (Pinctada martensii Dunker) by inhibiting the first polar body in eggs from triploids. J. Shellfish Res. 19, 147-151.

Hotta, M., Akira, K., Sousa, D., and Teixeira, J. (2017). Meiotic and early zygotic development in Crassostrea virginica observed through confocal microscopy. J. Shellfish Res. 36, 699-706. doi: 10.2983/035.036. 0319 bipolar spindles and some of the unclassified spindle patterns (Figures 1D-H,J,K).

\section{DATA AVAILABILITY STATEMENT}

The original contributions presented in the study are included in the article/supplementary material, further inquiries can be directed to the corresponding author/s.

\section{AUTHOR CONTRIBUTIONS}

YL and BZ conducted the experiments and wrote the manuscript. SL revised the manuscript. YG discussed with other authors about the experiment plan. All authors contributed to the article and approved the submitted version.

\section{FUNDING}

This study was partially supported by the National Key R\&D Program of China (2018YFD0901404), Tianjin Major Project of Seed Science and Technology (17ZXZYNC00020), Tianjin Modern Agro-Industry Technology Research SystemAquaculture-Shellfish Breeding Positions (ITTFRS2018046), Earmarked fund for Modern Agro-Industry Technology Research System (CARS-49), and Tianjin Natural Science Foundation (Grant No. 18JCQNJC84500).
Komaru, A., Matsuda, H., Yamakawa, T., and Wada, K. (1990). Meiosis and fertilization of the Japanese pearl oyster eggs at different temperature observed with a fluorescence microscope. Nip. Sui. Gak. 56, 425-430. doi: 10.2331/suisan. 56.425

Li, Y., Liang, J., and Xing, K. (2017). Preliminary study on tetraploid induction in Manila clam Ruditapes philippenarum with cytochalasin B. Fish. Sci. 36, 666-669.

Li, Y., Que, H., and Zhang, G. (2009). An immunofluorescent method for observation of meiotic apparatus of marine bivalves. Mar. Sci. 33, 38-40.

Longo, F., and Anderson, E. (1969). Cytological aspects of fertilization in the Lamellibranch, Mytilus edulis. I. Polar body formation and development of the female pronucleus. J. Exp. Zool. 172, 69-96. doi: 10.1002/jez.1401720107

Longo, F., and Anderson, E. (1970). An ultrastructural analysis of fertilization in the surfclam, Spisula solidissima. I. Polar body formation and development of the female pronucleus. J. Ultrastruct. Res. 33, 495-514. doi: 10.1016/s00225320(70)90177-2

Longo, F., Mathews, L., and Hedgecock, D. (1993). Morphogenesis of maternal and paternal genomes in fertilized oyster eggs (Crassostrea gigas): effects of cytochalasin B at different periods during meiotic maturation. Biol. Bull. 185, 197-214. doi: 10.2307/1542000

McCombie, H., Ledu, C., Phelipot, P., Lapegue, S., Boudry, P., and Gerard, A. (2005). A complementary method for production of tetraploid Crassostrea gigas using crosses between diploids and tetraploids with cytochalasin B treatments. Mar. Biotech. 7, 318-330. doi: 10.1007/s10126-004-0440-2

Muller-Reichert, T., Greenan, G., O’Toole, E., and Srayko, M. (2010). The elegans of spindle assembly. Cell. Mol. Life Sci. 67, 2195-2213.

Qin, Y., Xiao, S., Ma, H., Mo, R., Zhou, Z., Wu, X., et al. (2018). Effects of salinity and temperature on the timing of germinal vesicle breakdown and polar body release in diploid and triploid Hong Kong oysters, Crassostrea hongkongensis, in relation to tetraploid induction. Aqua Res. 21, 3647-3657. doi: 10.1111/are. 13833 
Que, H., Guo, X., Zhang, F., and Allen, S. (1997). Chromosome segregation in fertilized eggs from triploid Pacific oysters, Crassostrea gigas (Thunberg), following inhibition of polar body I. Biol. Bull. 193, 14-19. doi: 10.2307/ 1542732

Sluder, G., Miller, F., and Lewis, K. (1993). Centrosome inheritance in starfish zygotes II: selective suppression of the maternal in centrosome during meiosis. Dev. Biol. 155, 58-67. doi: 10.1006/dbio.1993. 1006

Stanly, J., Allen, S., and Hidu, H. (1981). Polyploidy induced in the American oyster, Crassostrea virginica, with cytochalasin B. Aquaculture 12, 1-10. doi: 10.1016/0044-8486(81)90002-8

Stephens, L., and Dowing, S. (1988). Inhibiting first polar body formation in Crassostrea gigas produces tetraploids, not meiosis triploid. J. Shellfish Res. 7, 550-551.

Tan, S. H. A., Teh, C. P., Chang, G. O., and Yasin, Z. (2017). Tetraploid induction in tropical oysters, Crassostrea belcheri (Sowerby) and Crassostrea iredalei (Faustino). Aqua Res. 48, 1406-1412. doi: 10.1111/are.12976

Yamamoto, S., and Sugawara, Y. (1988). Induced triploidy in the mussel, Mytilus edulis, by temperature shock. Aquaculture 72, 21-29. doi: 10.1016/00448486(88)90143-3

Yang, H., Que, H., He, Y., and Zhang, F. (2000a). Chromosome segregation in fertilized eggs from Zhikong scallop Chlamys Farreri (Jones \& Preston) following polar body I inhibition with cytochalasin B. J. Shellfish Res. 19, 101-105.

Yang, H., Zhang, F., and Guo, X. (2000b). Triploid and tetraploid Zhikong scallop, Chlamys farreri Jones et Preston, produced by inhibiting PB1. Mar. Biotech. 2, 466-475.

Yuan, J., Li, M., Wei, L. S., Yin, B., Xiong, S., Li, S., et al. (2009). Astrin regulates meiotic spindle organization, spindle pole tethering and cell cycle progression in mouse oocytes. Cell Cycle 8, 3384-3395. doi: 10.4161/cc.8.20.9885

Zhu, X., You, F., Zhang, P., and Xu, Y. (2008). Immunofluorescence observation on microtubule skeleton in turbot (Scophthalmus maximus) and flounder (Paralichthys olivaceus) eggs. Ocean Limnol. Sin. 39, 190-196.

Conflict of Interest: The authors declare that the research was conducted in the absence of any commercial or financial relationships that could be construed as a potential conflict of interest.

Copyright $(2021$ Li, Zhang, Liang and Guo. This is an open-access article distributed under the terms of the Creative Commons Attribution License (CC BY). The use, distribution or reproduction in other forums is permitted, provided the original author(s) and the copyright owner(s) are credited and that the original publication in this journal is cited, in accordance with accepted academic practice. No use, distribution or reproduction is permitted which does not comply with these terms. 